\title{
Pattern Recognition in Digital Images using Fractals
}

\author{
Mansoor Farooq, Mubashir Hassan Khan
}

\begin{abstract}
Pattern recognition in digital images is a conjoint problem with application in remote sensing, electron microscopy, medical imaging and astrophysics, still no general solution which can be rivalled with the human cognitive system in which a pattern can be conceded subject to random positioning and scale. This research has stemmed in the design and implementation of a new algorithm for general pattern recognition based on the use of fractal image compression. This approach has for the first time allowed the pattern recognition problem to be solved in a way that is invariant of rotation and scale. It allows both ANNs and correlation to be used subject to appropriate pre-and post-processing techniques for digital image processing.
\end{abstract}

Keywords: ANN, Cross-Correlation, Least Square Method, Fractal Image Compression and Pattern Recognition

\section{INTRODUCTION}

A digital image can be cogitated to be a visual display of a matrix of integers whose values define particular shades of grey, or specific color. The matrix or array epitomizes picture elements or pixels. Two hundred and fifty-six possible shades of grey can be portrayed in digital terms by a binary string of 128x128 binary digits ( 8 bits) either having a value zero or one. Such an exemplification view is known as "digitized" image and is suitable for processing by a digital computer. Image processing is a science that deals with image data. Image processing can be assumed as a distinctive form of two-dimensional processing used to unearth information about images. The methods of image processing can be applied to data even if the data is not evident. Image processing can be used to produce a visible image of purely numeric data enhanced in some manner to highlight some aspect of the data. For example, magnetic resonance medical imaging equipment, sonar, radar, ultrasound equipment, heat sensing equipment, fractals and so on. Aesthetics are not the only criterion by which to judge the effectiveness of the applied transformation. If the transformation is designed to bring out additional information and/or details not visible in the original image, the result can be considered successful even if it is not pleasing to look at. In this research we provided a solution to the problem in which it is hard to recognize a pattern if it has changed its orientation in the plane or scale.

Revised Manuscript Received on December 30, 2019.

* Correspondence Author

Mansoor Farooq*, Ph.D., Department of Computer Science and Engineering, Shri Venkateshwara University, Kashmir, India.

Mubashir Hussain Khan, Assistant Professor, Department of Computer Applications, Govt. College for Women M. A., Kashmir, India.

(C) The Authors. Published by Blue Eyes Intelligence Engineering and Sciences Publication (BEIESP). This is an open access article under the CC BY-NC-ND license (http://creativecommons.org/licenses/by-nc-nd/4.0/)

\section{ARTIFICIAL NEURAL NETWORKS}

Artificial Neural Networks (ANNs) is a powerful and practical tool for solving problems that would be difficult using conventional computer science techniques. The progress in the adaptation of Neural Networks as a problemsolving tool in the last few years is widespread, it has been proved successfully on a wide range of problems for object and pattern recognition (image recognition, speech recognition, etc.).

The three-layer neural network model has been widely implemented since it was discovered in 1980's and it is now one of the best-known Neural Network models [1], [2]. This three-layer ANN model has one input-layer with three nodes, one hidden-layer with one node and one-output-layer with three nodes

In this research we usefully connected three-layer ANN model with one input Layer with 64 nodes, one hidden-layer with 64 nodes and one-output-layer with 64 nodes, Back-Propagation algorithm network

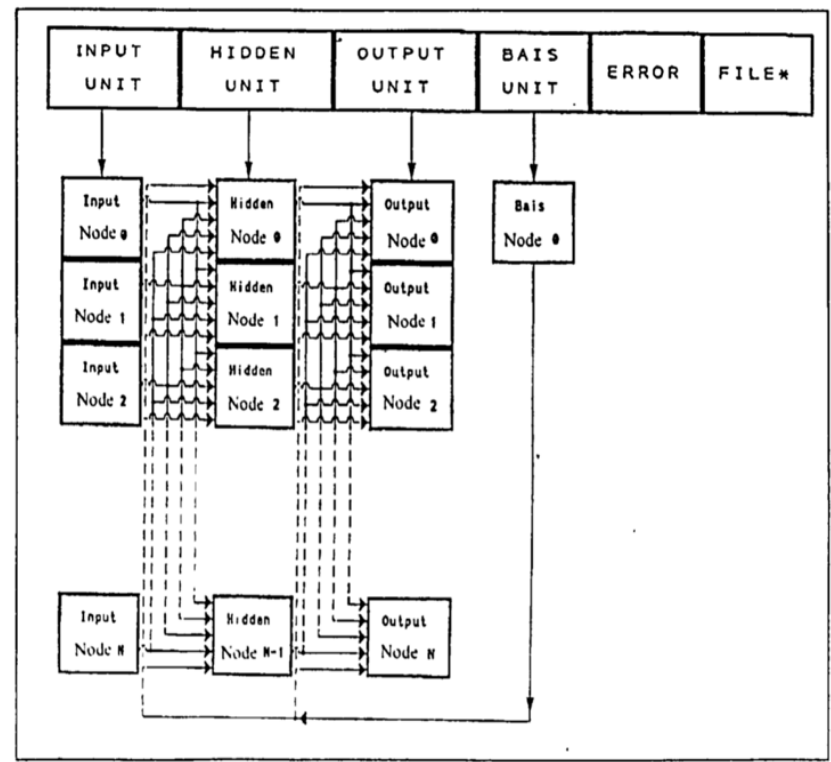

Fig. 1 The Artificial Neural Networks Model

The Neural Network was trained using the data compression method with a network on the un-prepossessed data image. $\mathrm{BP}$ is the most widely applied and successful algorithm for training stage. However, there is one problem related to the use of the BP algorithm and that is the time required to converge to small errors need a large number of patterns, especially for larger networks and complex pattern [3][4][5][7]. 


\section{A. The Back-Propagation Algorithm}

Learning problems occur in ANNs that are required to map a well-defined set of input units into a well-defined set of output units. They can generally be by the introduction of hidden units. The BP algorithm is the prescription originally suggested by Rumehart Hinton and Williams (1986) for dealing with the training of these hidden units [12]. The physical processes by a feedforward and feedbackward neural network architecture using the BP algorithm when successfully trained, maps the input parameters to the desired output. The neural network can learn into two different mode:

\section{The Incremental Mode}

In this mode the updating occurs after each pattern is presented i.e. update the input unit by multiplying the updating weight with output units.

\section{The Batch Model}

In this mode, updates increments are accumulated and updating takes place after all patterns have been presented.

The aim of the BP algorithm is to update the coupling weights in a neural network, i.e. to minimize the squared differences between the desired output (target) and the net output (Fig 2) shows the minimization function, where the $\mathrm{W}_{12}$ is the weight between the output and hidden layer, $\Delta \mathrm{W}_{12}$ is the new net output and $f_{j}(a)$ is the activation function.

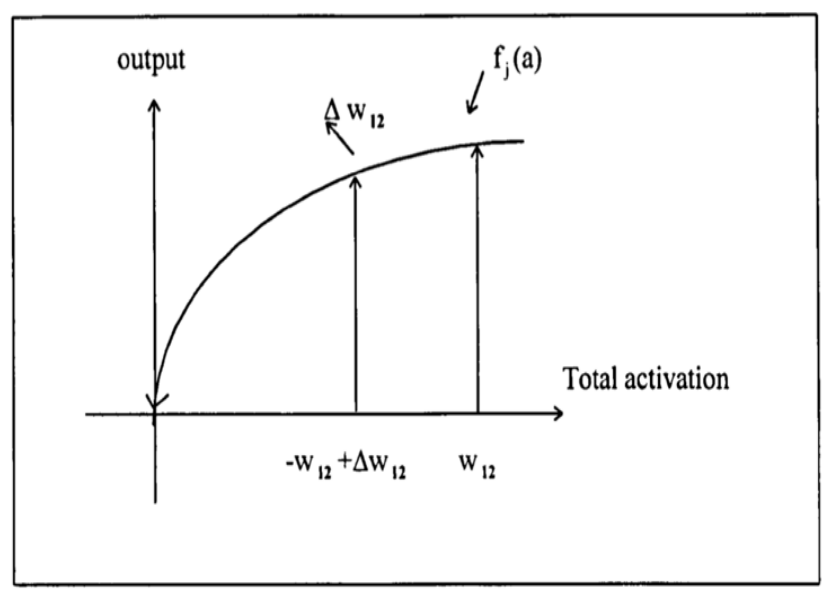

Fig. 2 The Minimization Function

\section{PROPOSED WORK AND METHODOLOGY}

\section{Fractal Transform Image Compression for Pattern Recognition}

Pattern recognition is increasingly being used in image processing for many fields such as handwriting, character, speech, remote sensing, motion detection recognition, etc.

There are many techniques used for automatic pattern recognition [1]. Artificial Neural Networks (ANN) is the most widely used technique for pattern recognition. Template matching, correlation or matching filtering are the methods used. These methods fail to recognise a pattern if it has changed its orientation in the plane or scale.

Here, we provide a solution to these problems by using a Fractal Image Compression technique. We applied generally used pattern recognition methods, which are (i) Cross-Correlation method and (ii) Least Squares method for comparison between these methods and the new solution.

\section{A.Cross-Correlation Method}

The Cross-Correlation applied generally used pattern recognition method is derived from the mean square error equation. If we assume that we have an input pattern $\mathrm{f}_{\mathrm{ij}}$, and an identically dimensioned template pattern $\mathrm{P}_{\mathrm{ij}}$, the matching (recognition) is obtained when the error is minimum.

Consider

$$
e=\sum_{i=0}^{N-1} \sum_{j=0}^{N-1}\left(f_{i, j}-p_{i, j}\right)^{2}
$$

Expanding this equation, we get

$$
e=\sum_{i=0}^{N-1} \sum_{j=0}^{N-1}\left(f_{i+k, j+1}^{2}-2 f_{i+k, j+1} p_{i+k, j+1}+p_{i+k, j+1}^{2}\right)
$$

$$
\begin{aligned}
& =\sum_{i=0}^{N-1} \sum_{j=0}^{N-1} \mathbf{f}_{i+k, j+1}^{2}-2 \sum_{i=0}^{N-1} \sum_{j=0}^{N-1} \mathbf{f}_{i+k, j+1} \mathbf{p}_{i+k, j+1}+\sum_{i=0}^{N-1} \sum_{j=0}^{N-1} \mathbf{p}_{i+k, j+1}^{2} \\
& \text { If we now assume that } \sum_{\mathbf{i}=\mathbf{0}}^{N-1} \sum_{j=\mathbf{0}}^{N-1} \mathbf{f}_{\mathbf{i}+\mathbf{k}, \mathbf{j}+1}^{\mathbf{2}} \text { and }
\end{aligned}
$$$$
1 \sum_{i=0}^{N-1} \sum_{j=0}^{N-1} \mathbf{p}_{i+k, j+1}^{2}
$$$$
\sum_{i=0}^{N-1} \sum_{j=0}^{N-1} \mathbf{f}_{i+k, j+1} \cdot \mathbf{p}_{i+k, j+1}
$$

are regular constants. Then the error is between the pattern $\mathrm{f}_{\mathrm{ij}}$ and the template pattern $\mathrm{P}_{\mathrm{ij}}$. Implementations and results using this method are illustrated in Fig. 3 where the square in the image on the left indicates where the correlation surface is a maximum.

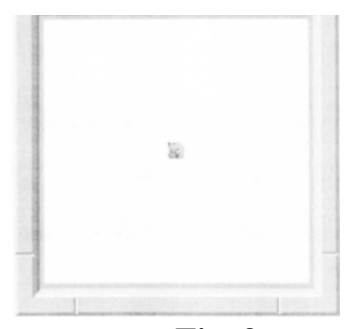

Fig. 3

Object Pattern

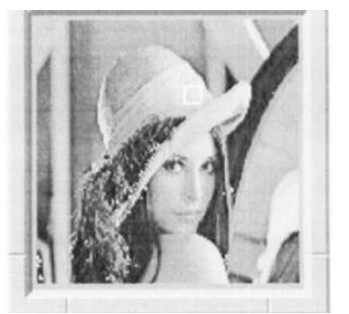

Fig. 3(a)

Pattern Recognition

\section{B. Least-Square Method}

Least-Square matching is perhaps the simplest pattern recognition technique. The patterns are identified by comparing the input pattern to a list of' stored pattern representations (templates). The principle is based on finding the minimum of the error function.

$$
\mathbf{e}=\sum_{\mathrm{i}=0}^{\mathrm{N}-1} \sum_{\mathrm{j}=0}^{\mathrm{N}-1}\left(\mathbf{f}_{\mathrm{i}, \mathrm{j}}-\mathbf{p}_{\mathrm{i}, \mathrm{j}}\right)^{2}
$$

Implementations and results using this approach are illustrated in Fig. 4 with different size of patterns where again the square in the image indicates the minimum of $\mathbf{e}$. 


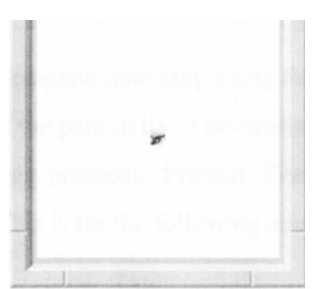

Fig. 4

Object Pattern

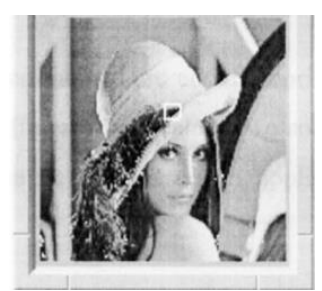

Fig. 4 (a)

Pattern Recognition

\section{Template Matching Using Fractal Image Compression Technique}

Template matching using the square or correlation method are not appropriate if the pattern has an orientation (rotated) or is a different size (scaled). To solve problem, Fractal Transform Image Compression has been applied.

This is for the following reasons:

1. By compressing the image, we work with a smaller size of data image hence we speed up the recognition operation.

2. The Fractal Transform Image Compression produces a readable compressed file unlike other compressors for example JPEG Compressor or Differential Image Compressor [5].

3. The Fractal transform copes with transformation by rotation, scaling and translation.

We have $\mathrm{L}^{1}$ distance [4] method illustrated in equation 3 used by the fractal transform image compression to solve the rotation variant and some scaling problem.

The L' distance method is used to find the matching input pattern $\mathrm{P}$ on the templates pattern $\mathrm{F}$ compressed data file. The L' method is used because it can be calculated faster and easier than the Hausdroff distance [3][4] L' and can be described as follows:

$$
L^{1}=\left|\sum_{i=0}^{N-1} \sum_{j=0}^{N-1}\left(f_{i, j}-p_{i, j}\right)\right|
$$

and if $\mathrm{L}^{\prime}$ is small (i.e. $\mathrm{L}^{\prime}=0$ ) then the two patterns (images) are alike.

4. Solution to the Rotation invariant Problem

The first and major problem for pattern recognition (i. e. rotation invariant) has been solved

\section{RESULTS AND DISCUSSION}

\section{Results of implementing the algorithm on Black and} White Images

The figures (5 to 9) shows the results of implementing the above algorithm on Black and White Images.

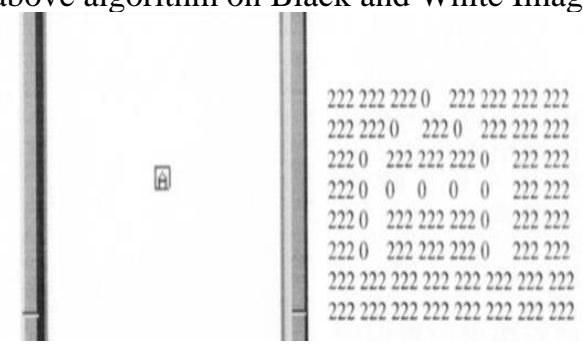

Fig. 5 A Block of Image Data Block Block
0029

00237

00165

00165

Compressed

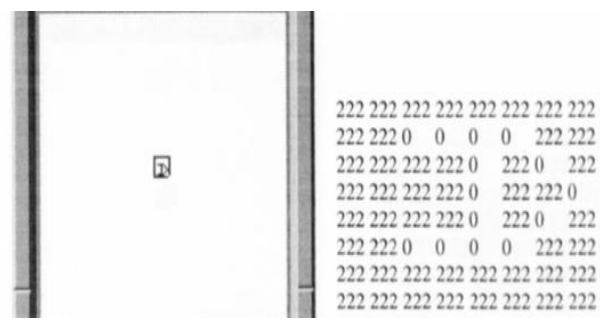

Fig. 6 Rotated Block $\left(90^{\circ}\right) \quad$ Data Block Compressed Block

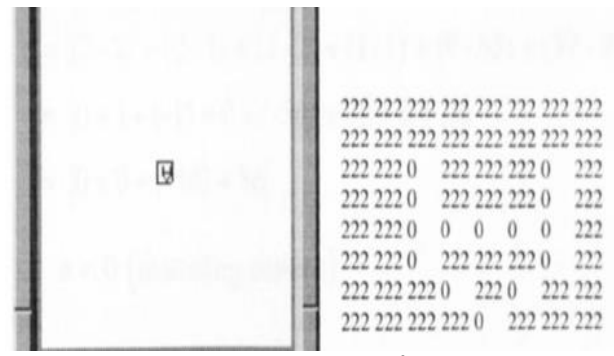

Fig. 8 Rotated Block $\left(270^{\circ}\right)$ Data Block Block

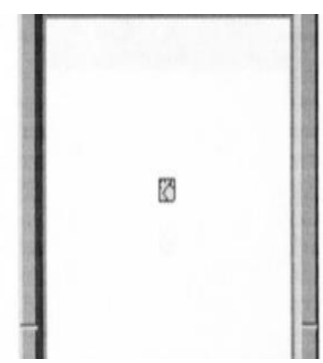

$\begin{array}{llll}0 & 2220 & 2222220 & 222222\end{array}$ $2222222222220 \quad 0 \quad 222222$ $\begin{array}{llll}0 & 2222220 & 2222220 & 222\end{array}$ $2222220 \quad 2222222222220$ $2220 \quad 222222222222222222$ $2222220 \quad 222222222222222$ $2222222220 \quad 222222222222$ $2222222222220 \quad 222222222$

Fig. 9 Rotated Block (450) Data Block Block

For example, applying the L1 matching equation between the original image 1 Fig. 5 and rotated block Fig. 6, we get $\mathrm{e}=\mid(2-2)+(2-1)+(1-2)+(1-1)+(9-65)+(37-9)+(65-65)$ $+(65-37)$

$$
\begin{aligned}
& =|0+1+(-1)+0+(-56)+28+0+28| \\
& =|0+0+(-56)+56| \\
& \text { Therefore } \mathrm{e}=0 \text { (matching pattern) }
\end{aligned}
$$

\section{Results of implementing the algorithm on Greyscale Images.}

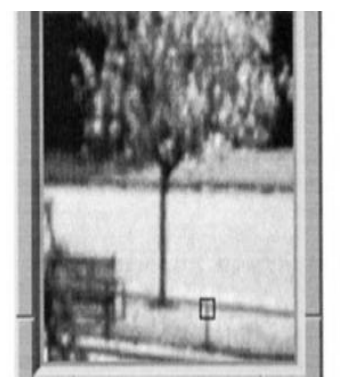

186190200210220220210208 156154162160174186182180 138138136114132138146150 168164150120122138136148 204192172124128164170174 202202174128136182192202 202202166136144200204206 202200172126150182202210

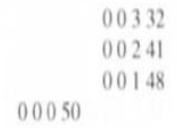

Fig. 10 A Block of Image Data Block Compressed Block 


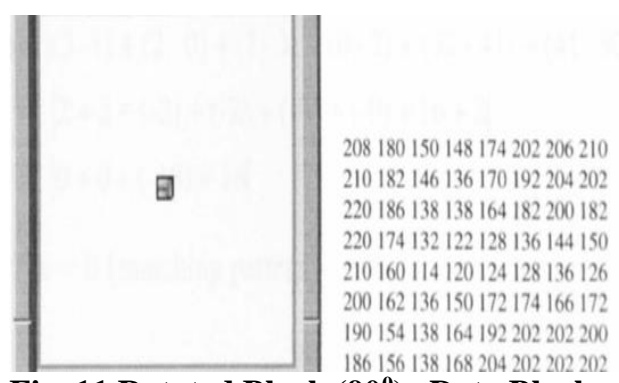

Fig. 11 Rotated Block $\left(90^{\circ}\right)$. Data Block Block

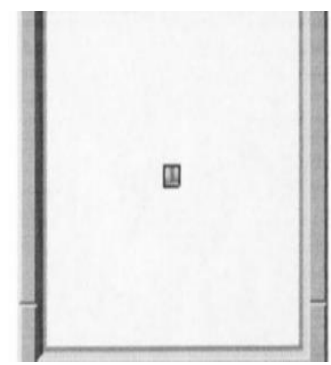

202200172126150182202210 202202166136144200204206 202202174128136182192202 204192172124128164170174 168164150120122138136148 138138136114132138146150 154154162160174186182180 186190200210220220210208

Fig. 12 Rotated Block (180) Data Block Compressed Block

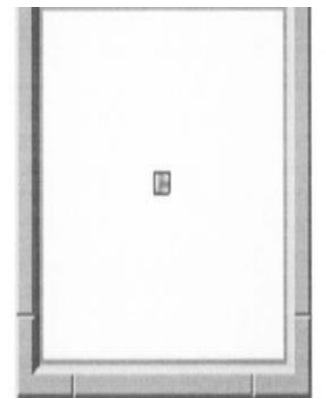

202202202204168138156186 200202202192164138154190 172166174172150136162200 126136128124120114160210 $150144136 \lcm{28} 122132174220$ 182200182164138138186220 202204192170136146182210 $210206202174|48| 50 \mid 80208$

\section{Fig. 13 Rotated Block (270º) Data Block Compressed Block}

For example, applying the L1 matching equation between the original image 1 Fig. 10 and rotated block Fig. 12, we get $\mathrm{e}=\mid(3-1)+(2-0)+(1-3)+(0-2)+(32-41)+(41-50)+(48-32)$ $+(50-48)$

$$
\begin{aligned}
& =|2+2+(-2)+(-2)+(-9)+(-9)+16+2| \\
& =|0+0+(-18)+18|
\end{aligned}
$$

Therefore $\mathrm{e}=0$ (matching pattern)

\section{CONCLUSION}

The Fractal Transform Image Compression technique developed has solved the rotation and scale variant pattern recognition problem. This method has been compared with different image compression algorithms such as JPEG, Block-Truncation-Coding, Differential Compression and Pixel Average Compression and pattern recognition methods such as Least Square method and Cross-Correlation method. The approach used for solving the pattern recognition problem has been fully explained and implemented. This solution opens the door to many real-world pattern recognition problems to be reconsidered such as fingerprint recognition, character recognition, signature recognition and medical image recognition etc.

\section{REFERENCES}

1. Blackledge J.M, (1993/94), C Programming, MSc Lecture Notes, Montfort University, School of Mathematical and Computing Science.

2. Blackledge J. M, (1993/94), Digital Image Processing, MSc Lecture Notes, Montfort University, School of Mathematical and Computing Science.

3. Lindley C.A, (1991), Practical Image Processing in C, John Wiley \& Sons, Inc, USA.

4. A. Roberts, M. Yearworth, Comparison of Preprocessing Transforms for Neural Network Classification of Character Images. Bristol Polytechnic, UK. PP 189, Image Processing and Its Application.

5. P. Flocchini, G. Mauri, F Gardin, MP Pensini, P. Stofella, Using Structured Input Patterns for Neural Based Image Recognition, Universita di Milano, Italy, PP 213, Image Processing and Its Applications.

6. G. D. Kendall, T. J. Hall, Performing Fundamental Image Processing Operations Using Quantified Neural Networks. King's College London, UK, PP 226, Image Processing and its Application.

7. Tian-Jin, Feug, Z. Honkes, M. J. Korsten, Internal Measuring Models in Trained Neural Networks for Parameter Estimation from Images, Ocean University, P R China, Twenty University, The Netherlands, PP 230, Image Processing and its Application.

8. Don Pearson, "Image Processing", 1991.

9. M. Barnsley, "Fractal Everywhere", Second Edition, 1993.

10. Yuval Fisher, "Fractal Image Compression, Theory and Applications", 1995.

\section{AUTHORS PROFILE}

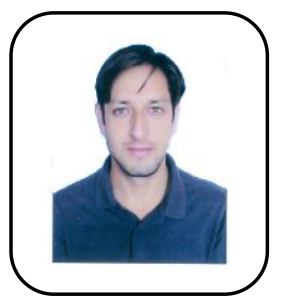

Mansoor Farooq, completed his Ph.D. in Computer Science and Engineering from Shri Venkateshwara University, MCA from Islamic University of Science and Technology and BCA from University of Kashmir, He has already published various research papers chapters and books. His core interest is image processing, Artificial Intelligence and IOT, besides that he is also a member of various professional computing societies like ACM New York, IAENG Hong Kong.

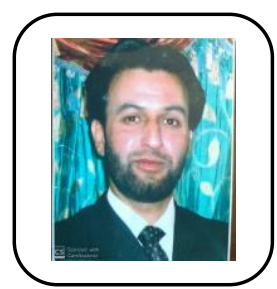

Mubashir Hussain Khan, Currently heading IT Section of Cluster University of Kashmir, Srinagar Previously worked as Assistant Professor Computer Applications, Govt College for Women MA, He has completed his masters MCA from University of Kashmir. His core interest includes IOT, Cloud Computing and Image Processing. 